\title{
Recovery of Nickel by Electrolytic Method from Electroplating Wastewater
}

\author{
Lei Zhu ${ }^{1, a}$, Jingsong Wang ${ }^{1, b,,}$, Zhengwei Xiong ${ }^{1, c,},{ }^{,}$, Qingwei Guo ${ }^{2, d}$,Weiming \\ Zhang ${ }^{3, e}$, and Ping Sun ${ }^{3, f}$ \\ ${ }^{1}$ School of Urban Construction, University of South China, Hengyang 421001 China \\ ${ }^{2}$ South China Institute of Environmental Science .MEP, Guangzhou 510632 China \\ ${ }^{3}$ School of the environment, Nanjing University, Nanjing 210046 China

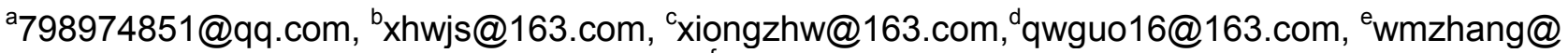 \\ nju.deu.cn, fsunping@nju.edu.cn
}

\begin{abstract}
Keywords:electrolytic method,recovery of nickel, electroplating wastewater, electrolytic parameters

Abstract: Electrolytic method is applied to recover nickel from the simulated nickel-containing electroplating wastewater. The experiment studied the main parameters affecting the recovery of nickel, including titanium plate, titanium mesh and graphite as cathode materials, other parameters such as initial concentration of nickel ions, voltage, plate distance, temperature and initial $\mathrm{pH}$ value. During the experiment, changing one parameter and keeping the other parameters constant for 6 hours and the recovery and current efficiency are used as evaluation indexes to describe the results of experiment. It's noticed that titanium plate is the optimum cathode and under the condition of initial concentration of nickel ions $20 \mathrm{~g} / \mathrm{L}$, voltage $4.0 \mathrm{~V}$, plate distance $15 \mathrm{~mm}$, temperature $50^{\circ} \mathrm{C}$ and initial $\mathrm{pH}$ value 7.0 , the recovery efficiency reaches the maximum of $73.12 \%$ and the current efficiency is $59.26 \%$.
\end{abstract}

\section{Introduction}

Electroplating is to use the principle of electrolysis plating a thin layer of the other metal or alloy in the surface of metal and electroplating industry is an important part of aircraft industry, automobile industry and metal processing industry ${ }^{[1-2]}$.According to the statistics, thousands of electroplating factories have been established in China and the annual amount of electroplating wastewater has reached 4 billion tons ${ }^{[3]}$. As only $30 \%$ to $40 \%$ of all the metals used in this process are effectively utilized $^{[4]}$, the electroplating wastewater contains a high heavy metal ions $\left(\mathrm{Cr}^{6+}, \mathrm{Cu}^{2+}, \mathrm{Ni}^{2+}, \mathrm{Zn}^{2+}\right)$ and they are highly toxic, carcinogenic. Without being treated, it will cause heavy metal pollution and resources waste ${ }^{[5-6]}$. Since nickel is cancerigenic and expensive ${ }^{[7]}$, therefore it's important to be treated before discharge and better to be recycled. This paper studied the main parameters affecting the recovery of nickel with electrochemical method ${ }^{[8]}$.

\section{Experiment}

Experimental Principle.Electrolysis is a process that make the current through the electrolyte solution or molten electrolyte cause redox reactions at the anode and cathode. Determined by its own electrochemical properties, $\mathrm{Ni}^{2+}$ can deposit at the cathode in the electrolysis process. The following chemical reactions will occur in the solution ${ }^{[9]}$ :

$$
\begin{gathered}
\text { Cathodic reaction: } \mathrm{Ni}^{2+}+2 \mathrm{e} \rightarrow \mathrm{Ni} \\
2 \mathrm{H}^{+}+2 \mathrm{e} \rightarrow \mathrm{H}_{2} \uparrow \\
\text { Anodic reaction: } 2 \mathrm{H}_{2} \mathrm{O} \rightarrow 4 \mathrm{H}^{+}+\mathrm{O}_{2} \uparrow+4 \mathrm{e}
\end{gathered}
$$

In this experiment, the main parameters affecting the effect of electrolysis are the anode material, initial concentration of nickel ions, voltage between the electrodes, plate distance, temperature of electrolyte, initial $\mathrm{pH}$ value.

Instruments and reagents. Instruments: $1000 \mathrm{~mL}$ breaker; $\mathrm{pH}$ meter(UB-7,Denver Instrument); electro-thermostatic water bath(DZKW-S-4,Shanghai Ke Heng Industrial Col.,Ltd);DC power 
(KXN-305D,Shenzhen Electronics Co., Ltd); atomic absorption spectrometer(AA6300,Japan); electrode plate: the titanium plate, titanium mesh or graphite is used as the anode and the cathode is stainless steel plate,the size of electrode is $80.00 \mathrm{~mm} \times 110.00 \mathrm{~mm} \times 2.00 \mathrm{~mm}$.

Reagents : $\mathrm{NiSO}_{4} \cdot 6 \mathrm{H}_{2} \mathrm{O}(\mathrm{AR})$ is used to make the simulated electroplating wastewater, $\mathrm{NaOH}$ and $\mathrm{H}_{2} \mathrm{SO}_{4}$ are used to regulate the $\mathrm{pH}$ value.

Schematic diagram of the self-made experimental device is shown in Fig.1.

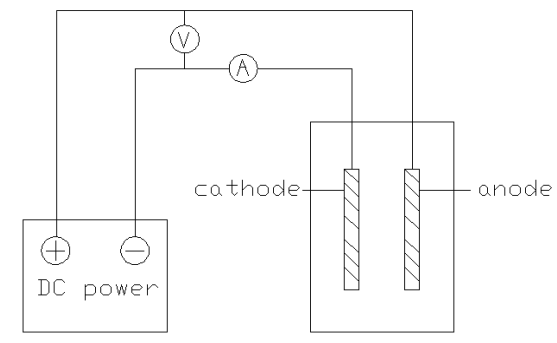

Fig.1 Schematic diagram of the self-made experimental device

Experimental Methods.Certain amount of simulated nickel-containing electroplating wastewater was put into the breaker. The electrolysis reaction persists for 6 hours under the condition of different parameters. Record the current value every hour, measure the residual concentration of nickel ions in solution and weigh the weight of deposit at the cathode after the electrodeposition. In this experiment, the recovery efficiency of nickel and the current efficiency are used as the evaluation indexes to describe the results of experiment. The calculation formulas are as followed:

$$
\begin{gathered}
\text { The recovery efficiency: } \eta_{1}=\frac{C_{0}-C_{1}}{C_{0}} \times 100 \% \\
\text { The current efficiency: } \quad \eta_{2}=\frac{\mathrm{b}}{I t k} \times 100 \%
\end{gathered}
$$

$\mathrm{C}_{0}$ is the initial concentration of nickel ions $(\mathrm{g} / \mathrm{L}), \mathrm{C}_{1}$ is the residual concentration of nickel ions $(\mathrm{g} / \mathrm{L})$, $\mathrm{b}$ is the weight of sediment at the cathode $(\mathrm{g})$, I is electric current $(\mathrm{A}), \mathrm{t}$ is electrolysis time $(\mathrm{h})$ and $\mathrm{k}$ is electrochemical equivalent $(\mathrm{g} /(\mathrm{A} \cdot \mathrm{h}))$.In this experiment, the electrochemical equivalent of nickel is $1.095 \mathrm{~g} /(\mathrm{A} \cdot \mathrm{h})$.

\section{Results and Analysis}

Effect of anode material.Under the condition of voltage $4.0 \mathrm{~V}$,temperature $40^{\circ} \mathrm{C}$, plate distance $20 \mathrm{~mm}$, the initial concentration of nickel ions $20 \mathrm{~g} / \mathrm{L}$ and initial $\mathrm{pH}$ value 5.0 , the effect of three different kinds of anode materials are tested. The result showed in Fig. 2 reveals that e the recovery efficiency of the three anodes all rise as the electrolytic time continue. The anode material need to meet the requirements of good conductivity, strong corrosion-resisting, long work life and insolubility. In the case of titanium plate for the anode, the recovery efficiency is the highest. It's may because the titanium plate is harder to be passivated and its electrical conductivity is better than titanium mesh and graphite. It suggests that titanium plate is the optimum anode material.

Effect of initial concentration of nickel ions. Within a certain range, with the increase of concentration of nickel ions, the reduction potential of nickel increase and overpotential of nickel decrease, which is good for the reduction of $\mathrm{Ni}^{2+}$ in the cathode. But with the nickel concentration continue to increase, the overpotential of nickel increases and the reduction potential of nickel stays on a low level. At the same time, the reduction of $\mathrm{H}^{+}$become much more competitive, which leads to the recovery of nickel to be harder.

Under the condition of voltage $4.0 \mathrm{~V}$,temperature $40^{\circ} \mathrm{C}$, plate distance $20 \mathrm{~mm}$, initial $\mathrm{pH}$ value 5.0 and titanium plate as the anode, change the initial concentration of nickel ions $(10 \mathrm{~g} / \mathrm{L}$ to $60 \mathrm{~g} / \mathrm{L})$ to analysis the results presented in Fig.3. When the concentration of nickel ions increases from $10 \mathrm{~g} / \mathrm{L}$ to $20 \mathrm{~g} / \mathrm{L}$, the recovery of nickel increases rapidly and as the concentration continue to increase, the removal rate increases slowly and the current efficiency is reducing. Compared comprehensively, the proper initial concentration of nickel ions is $20 \mathrm{~g} / \mathrm{L}$. 


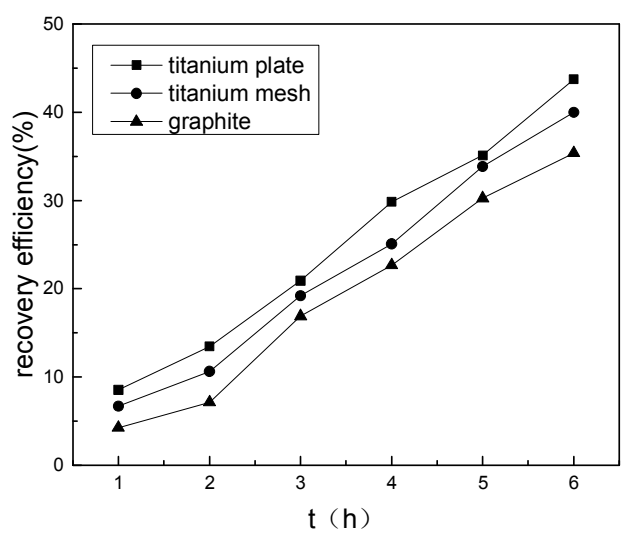

Fig.2 Effect of anode material

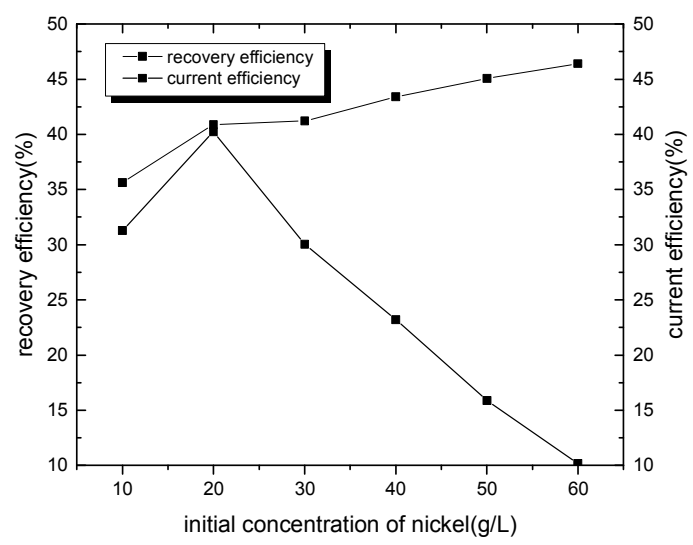

Fig.3 Effect of initial concentration of nickel ions

Effect of temperature of electrolyte.Temperature is one of the important parameters affecting electrochemical reaction kinetics. Temperature rise will cause the activation energy to reduce and diffusion coefficient to increases. On the other hand, the temperature rise will sped up the rate of ions diffusion and the deposition rate of $\mathrm{Ni}^{2+}$. But at the same time, high temperature means high energy consumption, which is an important economic factor that needed to be considered.

Under the condition of voltage $4.0 \mathrm{~V}$, the initial concentration of nickel ions $20 \mathrm{~g} / \mathrm{L}$, plate distance $20 \mathrm{~mm}$,initial $\mathrm{pH}$ value 5.0 and titanium plate as the anode to test the affect of temperature on recovery of nickel and the result is showed in Fig.4. We can see the recovery and current efficiency all rise as the temperature goes up. But the current efficiency increases slowly after $50^{\circ} \mathrm{C}$,so the optimal temperature is $50^{\circ} \mathrm{C}$.

Effect of voltage between the electrodes.In the case of plate distance is constant, the bigger the voltage, the greater the electric field intensity between the plates. So in the electrolysis higher voltage is advantageous to the precipitation of nickel.

Under the condition of temperature $50^{\circ} \mathrm{C}$, the initial concentration of nickel ions $20 \mathrm{~g} / \mathrm{L}$, plate distance $20 \mathrm{~mm}$, initial $\mathrm{pH}$ value 5.0 and titanium plate as the anode. Change the voltage from $2.0 \mathrm{~V}$ to $5.0 \mathrm{~V}$ to see the effect of voltage on the recovery of nickel. As is showed in Fig.5, the recovery and current efficiency all go up with the increasement of voltage. But when the voltage $\geqslant 4.5 \mathrm{~V}$, the power supply is unable to maintain the constant voltage, which is damage to the test condition and lots of energy is needed. It can be explained as follows: the increasement of voltage will accelerate the remove of ions between the plate, so it can promote the reduction of $\mathrm{Ni}^{2+}$. When the voltage continue to rise, it will also accelerate the reduction of $\mathrm{H}^{+}$at the cathode to compete with $\mathrm{Ni}^{2+}$.Accordingly, the optimum voltage is $4.5 \mathrm{~V}$.

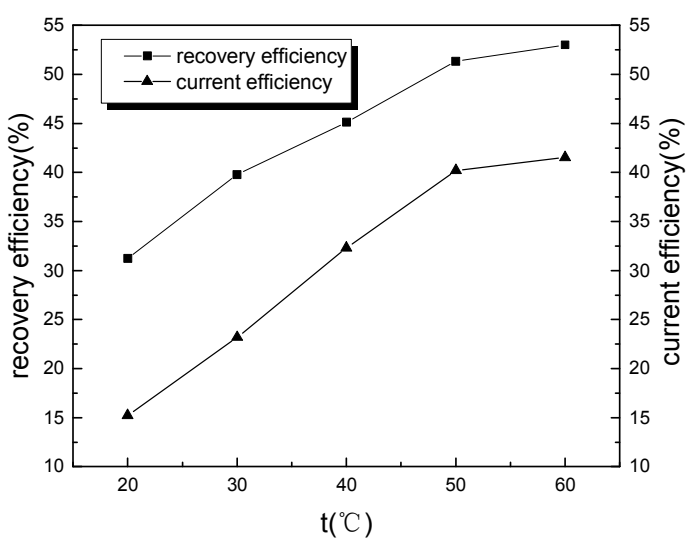

Fig.4 Effect of temperature of electrolyte

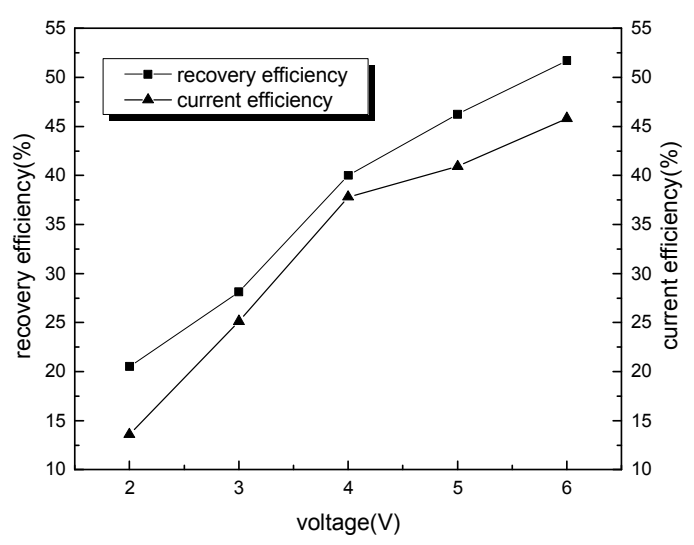

Fig.5 Effect of voltage between the electrodes 


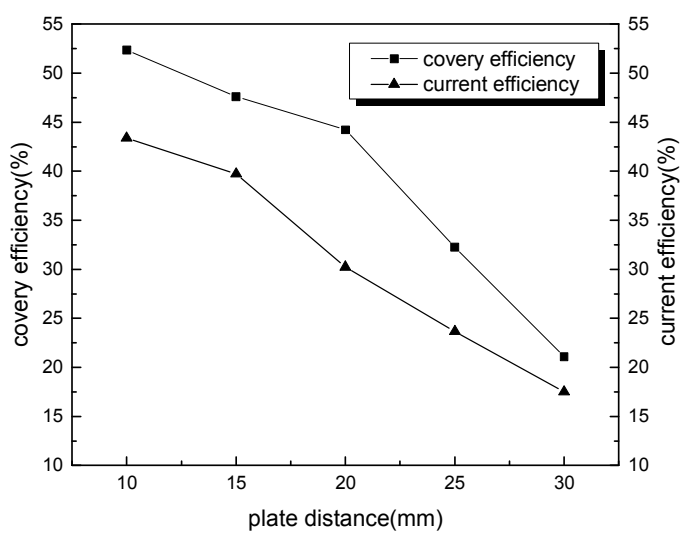

Fig.6 Effect of plate distance

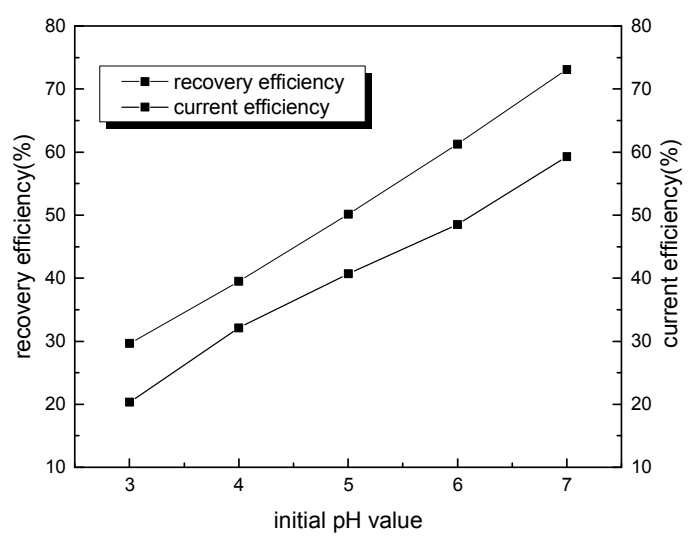

Fig.7 Effect of initial $\mathrm{pH}$ value

Effect of plate distance. With the increase of the plate distance, the current density of electrolyte decreases, the number of electron out of the cathode reduce in unit time and the impedance value of reduction reaction increases, which will all lead to the decrease of recovery and current efficiency. On the other side, smaller plate distance will result in current path.

Under the condition of voltage $4.5 \mathrm{~V}$,temperature $50^{\circ} \mathrm{C}$, the initial concentration of nickel ions $20 \mathrm{~g} / \mathrm{L}$, and titanium plate as the anode, change the plate distance from $10 \mathrm{~mm}$ to $30 \mathrm{~mm}$ to do the experiment. From the results presented in Fig.6,it can been seen that with the increasement of plate distance, both the recovery and current efficiency decease, it suggests that smaller distance is conducive to the recovery of nickel, but it will also leads to the formation of current path between the plate and the current increases sharply, which finally will be harmful to the power supply. Considering various influences, the appropriate distance is $15 \mathrm{~mm}$.

Effect of initial pH value.pH is an important parameter in the metal deposition. It will influence the hydrogen discharge, stress and hardness in the deposit and the cathode and anode efficiencies. The initial $\mathrm{pH}$ value of solution is varied by $\mathrm{NaOH}$ and $\mathrm{H}_{2} \mathrm{SO}_{4}$.

Under the condition of voltage $4.5 \mathrm{~V}$,temperature $50^{\circ} \mathrm{C}$, plate distance $15 \mathrm{~mm}$ the initial concentration of nickel ions $20 \mathrm{~g} / \mathrm{L}$ and titanium plate as the anode, change the initial $\mathrm{pH}(3.0$ to 8.0$)$ to analysis the effect of the initial $\mathrm{pH}$. The results presented in Fig. 7 showed as the $\mathrm{pH}$ increases, the recovery and current efficiency increase, but when the $\mathrm{pH}>7.0$ the recovery decreases rapidly. It's because when the initial $\mathrm{pH}$ value at a low level, electro-chemical polarization and concentration polarization causes the reduction of $\mathrm{H}^{+}$to rise and the reduction of $\mathrm{Ni}^{2+}$ to be suppressed. When the $\mathrm{pH}>7.0$, the nickel begins to precipitate as nickel hydroxide. So the optimum initial $\mathrm{pH}$ value is 7.0.

\section{Conclusions}

Electrolysis is an environmentally friendly technology, it's high efficiency, low-cost, manageable and has the function of air flotation, flocculation and sterilization. It could produce purity reclaimed nickel, and the organic compound could be effectively removed through electrolysis. In general, the initial concentration of nickel ions in electroplating wastewater is below $20 \mathrm{~g} / \mathrm{L}$, so before the electrolysis the nickel-containing wastewater need to be concentrated ion exchange technique.

The experiment proves that using the electrolytic method to recover nickel from the simulated nickel-containing electroplating wastewater is feasible. Using the titanium plate as the anode, the experiment carried out for 6 hours under the condition of the initial concentration of nickel ions $20 \mathrm{~g} / \mathrm{L}$, temperature $50^{\circ} \mathrm{C}$, voltage $4.0 \mathrm{~V}$, plate distance $15 \mathrm{~mm}$ and initial $\mathrm{pH}$ value 7.0 , finally the recovery efficiency reaches the maximum of $73.12 \%$ and the current efficiency is $59.26 \%$.

\section{Acknowledgments}

This research was financial supported by the Water Special Program of China's 12th Five-year Plan (2012ZX07206-003) and the Scientific and Technological Innovation project of University of 
South China(NHCXTD07) .

\section{References}

[1] Wen-juan Zhang,Ling Liu, Cheng-mei Li.Industrial Safety and Environmental Protection, Vol.32(2006),P.35(in Chinese)

[2] Wen-xing Wang. Plating and Finishing, Vol.33(2011),P.42(in Chinese)

[3] F L Fu, Q Wang. Journal of Environmental Management,Vol.92(2011),P.407

[4] M. Zhao, J. R. Duncan, and R. P. Van Hille.WaterResearch,Vol.33(1999) , P.1516

[5]R S Juang,H C Kao and W Chen.Separation and Purification Technology,Vol.49(2006),P.36

[6] Srivastava NK,Majumder CB. Journal of Hazardous Materials, Vol.151(2008),P.1

[7] L Koene,L J Jassen. Electrochemical Acta. Vol.47(2001), P.695

[8] X J Yu, D Zhou, H Y Wang. Acta Scientiae Cirumstantiae. Vol.20(2000),P.120

[9] Ying-chun Lei. Urban Environment \& Urban Ecology. Vol.22(2009), P.13(in Chinese) 